\title{
DESPENALIZACION DE LA EUTANASIA, DERECHO A UNA VIDA DIGNA, LIMA-2019.
}

\section{DEPENALIZATION OF EUTANASIA. RIGHT TO A LIFE WITH DIGNITY, LIMA-2019.}

Delia CHÁVEZ SANTAMARÍA

\author{
Dr. Jorge RODRIGUEZ FIGUEROA
}

RESUMEN. El presente artículo se efectúa con el fin de definir si la despenalización del homicidio piadoso es el mecanismo jurídico para el reconocimiento del derecho a poder llevar una vida digna a través de elegir morir dignamente en casos personas diagnosticadas con enfermedades terminales que les causen dolores insoportables. Con respecto a la información recopilada, relacionada al tema de investigación, la primera parte de este trabajo hace referencia a la eutanasia y los países pioneros en legalizarla, así como se hace mención al proyecto de Ley presentadlo y que fue rechazado por el congreso, de la misma manera en la primera parte de este trabajo de investigación se desarrolla brevemente los tipos de eutanasia. Por otro lado, también veremos lo que opinan otros países respecto a la legalización de la eutanasia.

Posteriormente a desarrollar las teorías relacionadas a la eutanasia, se elabora la formulación del problema de investigación y la justificación del estudio realizado, así mismo, se determinó los objetivos tanto generales como específicos, luego se identificó los instrumentos de estudio que se utilizará para determinar si la despenalización del homicidio piadoso es el medio jurídico para reconocer el derecho a morir dignamente como sustento de una vida digna.

ABSTRACT. This article is carried out in order to define whether the decriminalization of pious homicide is the legal mechanism for the recognition of the right to lead a dignified life through choosing to die with dignity in cases diagnosed with terminal illnesses that cause them pain unbearable.

With respect to the information collected, related to the research topic, the first part of this work refers to euthanasia and the pioneer countries in legalizing it, as well as mentioning the draft law presented and which was rejected by the congress, the same way in the first part of this research work the types of euthanasia are developed briefly. On the other hand, we will also see what other countries think about the legalization of euthanasia.

After developing the theories related to euthanasia, the formulation of the research problem and the justification of the study carried out are elaborated; Likewise, the general and 
specific objectives were determined, then the study instruments were identified that will be used to determine if the decriminalization of pious homicide is the legal means to recognize the right to die with dignity as a support for a dignified life.

Palabras Claves: Eutanasia, vida digna, enfermedad terminal, homicidio piadoso.

Keywords: Euthanasia, dignified life, terminal illness, pious homicide.

\section{RESUMEN}

El presente artículo tiene como objetivo demostrar que la eutanasia es el medio por el cual los pacientes diagnosticados con enfermedades terminales, pueden hacer valer su derecho a la dignidad, no solo a vivir dignamente, sino a morir con dignidad, así mismo, a que se respete su derecho a elegir si quiere continuar viviendo con los intensos dolores que son provocados por su enfermedad o si quieren darles fin a ellos.

En ese sentido es preciso entender que la eutanasia va más allá de darle fin a una vida, como lo ven muchas personas e incluso la religión, la eutanasia es un mecanismo existente con la finalidad de calmar el sufrimiento de los enfermos terminales, quienes ven en este procedimiento un alivio al dolor que padecen día a día y que no les permite el desarrollo de su dignidad y el derecho a la libertad que se ven vulnerados a través, de la penalidad de este mecanismo tal como lo prescribe el Art. 112 del Código Penal Peruano quien lo tipifica como Homicidio Piadoso.

En ese sentido, el presente artículo tiene como finalidad demostrar la necesidad de la despenalización de la eutanasia a fin de que sea aplicada a los enfermos terminales que la soliciten.

Como ya se ha explicado la problemática radica en la vulneración de los derechos fundamentales del que gozamos todos, tales como, el derecho a la dignidad y derecho a la libertad. 
Cabe indicar que el presente artículo sigue las etapas que debe incluir el informe de un artículo científico, las cuales son:

I. Introducción

II. Metodología

III. Resultados

IV. Discusión y conclusión

V. Bibliografía

A continuación, se exponen cada una de las etapas antes mencionadas.

\section{INTRODUCCIÓN}

La eutanasia fue considerada una crisis en las sociedades primitivas en que se ponía en práctica dar muerte a las personas que se las creía inútiles para la sociedad, esta costumbre fue aceptada socialmente y se practicaba a los recién nacidos con malformaciones, hasta la llegada del cristianismo quien prohibió esta práctica, hasta el siglo $\mathrm{XX}$ en que algunos países pretenden su legalización.

En el Perú, el código penal en su artículo 112 del C.P. señala lo siguiente: "El que por piedad mata a un enfermo incurable que le solicita de manera expresa y consciente para poner fin a sus intolerables dolores, será reprimido con pena privativa de libertad no mayor de tres años", como antecedente el año 2015 el Congresista Roberto Angulo Álvarez a través del grupo parlamentario Dignidad Democrática presentó ante el Congreso el P.L. $\quad N^{o} \quad 4215-2015-C R$ Ley que despenaliza el homicidio piadoso, proyecto que no fue aprobado, pese a ello considero que la despenalización de la eutanasia hoy en día es un tema que debemos tratar a fin de lograr su aplicación, toda vez que, el homicidio piadoso es una necesidad para los enfermos incurables que padecen de dolores insoportables, quienes no quieren ver su dignidad como personas, menoscabada por sus enfermedades, y dicho problema no es un tema de derecho, sino un tema social que sufren las grandes sociedades en la actualidad.

Algunas de las causas de este problema es demostrar el porqué de la necesidad de la despenalización del homicidio piadoso más conocido como eutanasia, hoy en día se manifiesta como un tema controversial, como se ha podido apreciar de diversos casos que se mencionaran como ejemplo de otras jurisprudencias extranjeras. Si 
bien es cierto el tema es muy controvertido, es un tema al que siempre llega tanto el profesional en derecho como el profesional en medicina, sin embargo, se enfocara en lo concerniente al Derecho, toda vez que, el mayor problema de la despenalización de la eutanasia es penado, la acción de dar muerte a una persona con enfermedad terminal y que sufre, haciendo prevalecer el derecho a la dignidad humana o de vivir digno. De mantenerse este problema, desde el punto de vista Epistemológico, como lo señala Silverman (2000, p. 89) afirma que la observación es fundamental para entender otra cultura y por ende el fenómeno estudiado del hecho de pensar que se da muerte a una persona intencionalmente, no podrá verse el verdadero fondo o significado de la eutanasia que no es el simple hecho de quitar la vida, sino de aplacar el dolor y el padecimiento tanto del paciente así como de los familiares, toda vez que con la aplicación de eutanasia no solo es el paciente, que se beneficia al tener una muerte digna, también son sus familiares quienes ya no estarán estancados cuidando del paciente y muchas veces truncando sus proyectos de vida, de otro lado es el estado quien debe decidir que los profesionales en base a las necesidades y realidades apliquen la eutanasia ya que dejara de subsidiar los gastos que implica mantener a un paciente en las condiciones críticas ya sea por el costo de los medicamentos, gastos clínicos entre otros. La Industria farmacéutica debe, ser quien confirme que la vida digna es en un estado que contradice al menoscabo y sufrimiento de una enfermedad terminal, dejando de lado sus intereses económicos.

La eutanasia en el Perú, no solo implica modificar nuestro Código Penal, sino también, las normas que rigen el comportamiento médico, toda vez que, los médicos tienen como principal función velar por el bienestar de su paciente lo que ellos entienden alargar la vida de los pacientes lo más que puedan, ¿pero para el paciente esto es vida?, por lo que, este tema no solo atañe al ámbito medico sino jurídico y también implicancias en el ámbito social, cultural, económico, que desde la dación del código napoleónico, no existe modificaciones en las normas adecuadas a 
la realidad social. Porque, en el presente problema sufre la víctima y sus familiares $\mathrm{y}$ el estado sin fundamento alguno sanciona penalmente y las Industrias farmacéuticas lucran en esta situación que es irreversible para los familiares y la víctima.

El problema en la investigación científica es un vacío legal, que debe ser abordado por los no solo por el derecho sino por las demás disciplinas, conforme lo señala Huamanchumo y Rodríguez (2015).

A fin de fortalecer el presente artículo, se investigó el tratamiento que se le da a la eutanasia en otros países habiendo obtenido la siguiente información:

Asia Pacific Journal of Health Law \& Ethics, 2019. Responding to Calls to Legalize Euthanasia and Physician Assisted Suicide in Singapore.

El estudio refiere a que no existe mayor estimulo moral que la necesidad de aplacar el padecimiento de las personas diagnosticadas con enfermedades incurables a partir de la aplicación de la eutanasia o el suicidio asistido por personal de salud, llámese médico o en algunos casos enfermeras, convirtiéndose en un derecho de un paciente autónomo con enfermedad terminal, pese al estudio realizado, sigue siendo ilegal la eutanasia, sin embargo, se sigue luchando para influir en el sentir del pueblo con la finalidad de conseguir la legalización de la eutanasia.

Thomas Morrow, 2019. Voluntary Euthanasia, Assisted Suicide \& Law Reform: A Sketch of the Canadian Experience.

El estudio concluyó que no es posible justificar la prohibición total de la eutanasia, toda vez que las prácticas de darle fin a la vida ya son legales en Canadá, sim embargo lo que no se encuentra permitido es que a través de los testamentos en vida se busque solicitar una aplicación de la eutanasia, toda vez que para que esta opere es indispensable el consentimiento del individuo a fin de garantizar la legalidad del procedimiento.

Barbara Pesut, 2019. Medical Assistance in Dying: A Review of Canadian Nursing Regulatory Documents.

El estudio señala que desde que se legalizó la muerte asistida el año 2016, esta ha tenido un alto índice de aceptación 
sobre todo en los pacientes provenientes de las zonas urbanas en Canadá, siendo que en tan solo un año hubo 1961 muertes bajo el procedimiento de muerte asistida, siendo a la fecha de investigación un total de 3714 muertes asistidas desde su legislación, provocando que los profesionales encargados de asistir dicha práctica, se sientan cada vez que brindan una ayuda a otra persona, razón por la que la Carta Canadiense de Derechos y Libertades garantiza los procedimientos que conlleven a la práctica de la eutanasia.

Robert Preston 2018. Death on demand? An analysis of physician-administered euthanasia in The Netherlands.

El estudio concluye que para legalizar la eutanasia en Holanda (Países Bajos) su principal fundamento fue el hecho de atenuar el padecimiento que padecen los enfermos, en base a dicho fundamento, se determinó que la eutanasia es el medio legal para evitar que los individuos que adolecen enfermedades crónicas que pueden causarles años de dolor se vea sopesado no solo a las personas que tienen el padecimiento sino a sus familiares quienes también se ven afectados.

Sigrid Dierickx, Luc Deliens, Joachim Cohen and Kenneth Chambaere, 2018. Involvement of palliative care in euthanasia practice in a context of legalized euthanasia: A population-based mortality follow-back study.

El estudio concluye que, en el contexto de la legalización de la eutanasia, esta y los cuidados paliativos guardan relación entre sí, toda vez que cierta cantidad de personas atendidas en los centros de cuidados paliativos solicita la eutanasia, siendo positiva la respuesta a esta solicitud. Sin embargo, el mismo estudio propone que existe la posibilidad que el personal de salud que trabaja en los centros de cuidados paliativos contrapone sus actitudes con sus ideas personales, toda vez que, el deseo de ayudar a las personas a morir y de esta manera aliviar su sufrimiento sobrepasa al hecho de ayudar a vivir a una persona cuya vida ya no tiene mucha esperanza.
Abohaimed, Matar, Al-Shimali, Al Thalji, Al-Othman, Zurba and Shah, 2018. 
Attitudes of Physicians towards Different Types of Euthanasia in Kuwait.

El estudio realizado concluye que el Ministerio de Salud de Kuiwait debe capacitar al personal de salud sobre la eutanasia y sus tipos, toda vez que si bien es cierto las dos quintas partes de los médicos manifestaron estar a favor de la eutanasia, este tema no es muy abordado por las autoridades correspondientes, ocasionando que no exista un protocolo a seguir en situaciones en las que la familia o pacientes solicitan la eutanasia, hecho que podría estar ocurriendo en nuestra sociedad, toda vez que, nuestras autoridades no informan sobre el uso o aplicación de la eutanasia, por el contrario muchas de ellas se encuentran en contra de su aplicación quizá por falta de información.

Barbuzzi, M. (2014). Who Owns the Right to Die? An Argument about the Legal Status of Euthanasia and Assisted Suicide in Canada. Penn Bioethics Journal, 10 (1) 16-20

El estudio concluye que para el sistema legal Canadiense no se considera la calidad de vida como fundamento valido para la permisión de la eutanasia y el suicidio asistido, sin embargo, se considera la calidad de vida como un factor decisivo en el ámbito legal, ya que para el paciente que solicita la eutanasia a nivel personal considera que su deseo de invocar o hacer valer su derecho de suspender todo tipo de tratamiento parte desde la perspectiva del deterioro de su calidad de vida, siendo que al aprobar suspender cualquier tipo de tratamiento se está respetando la libertad del paciente y no el derecho a morir.

Chan, B., \& Somerville, M. (2016). Converting the "Right to Life" to the "Right to Physician-Assisted Suicide and Euthanasia": An Analysis of Carter v Canada (Attorney General), Supreme Court of Canada.

El estudio realizado respecto al fallo de la Corte Suprema de Canadá, concluye que el citado fallo se debe interpretar como una victoria tanto en el aspecto legal como en el médico, toda vez que, la modificación de la Ley permite que los médicos apliquen la eutanasia a sus pacientes, cabe resaltar que como en el caso anterior, Canadá es un país que tiene respeto por la vida de los demás, pero que 
también respeta la autonomía individual, hecho que se debe tener en cuenta, ya que nuestra legislación ampara nuestra libertad de decisión, así como nuestra dignidad.

Latham, S. R. (2015). "Aid in dying" in the courts. The Hastings Center Report, 45(3), 11-12.

El artículo señala que en algunos estados de los Estados Unidos no se encuentran permitidos la asistencia al suicidio ni la eutanasia, sin embargo, en dos estados en particular en los que no es legal la ayuda al suicidio, ha habido casos en los que el Tribunal falla a favor creando incertidumbre legal sobre el tema, toda vez que, al existir un fallo judicial abalando la ayuda al suicidio, se presta a que se realice dicha práctica sin tener en cuenta ningún procedimiento, es por ello ya importancia de legalizar la eutanasia a fin de evitar conflictos legales y se realice todo el procedimiento dentro del marco legal.

Shibata, B. (2017). An Ethical Analysis of Euthanasia and Physician-Assisted Suicide: Rejecting Euthanasia and Accepting Physician Assisted Suicide with Palliative Care. Journal of Legal Medicine, 37(1/2), 155-166.

El estudio concluye que la asistencia a morir a diferencia de la eutanasia, es que la asistencia a morir te permite protegerte del abuso, y asegura una decisión autónoma del paciente, toda vez que, en la muerte asistida es el paciente quien por sus propios medios da fin a su vida, con asesoramiento médico, para lo cual antes de llegar a esta decisión, los médicos sugieren a los pacientes que antes de acogerse al suicidio asistido, someterse a cuidados paliativos, decisión que queda en manos del paciente, a diferencia de la eutanasia que es el medico quien realiza todo el procedimiento.

\section{METODOLOGÍA}

Respecto a los aspectos metodológicos se mencionarán los siguientes:

\section{a) Tipo Y Diseño:}

El diseño de la investigación se basa en la teoría fundamentada el cual, es un diseño metodológico que pretende generar teorías que explican un fenómeno social en su contexto natural, en este trabajo de investigación el fenómeno social de 
estudio es la aplicación de la eutanasia por ser de interés público, siendo la presente investigación más interpretativa, por lo que a través de información recabada sobre la legalización de la eutanasia en otras legislaciones se podrá demostrar que en nuestro país es posible llegar a legalizar dicha práctica siempre que esta sea realizada por un médico.

Para Strauss \& Corbin (2002) la teoría fundamentada tiene como función identificar los procesos sociales básicos, siendo que en la presente investigación se estudiara si es posible legalizar la eutanasia en el Perú, a través de esta metodología se podrá identificar qué aspectos son relevantes para lograr la despenalización de la eutanasia. (p. 15)

La finalidad el presente método es, la vida, la relación paciente y médico y la libertad a vivir una vida digna. En Colombia Gustavo Quintana, es conocido como el "Doctor Muerte", quien hizo más de 200 eutanasias en 35 años, nos preguntamos cuantas familias dejaron de sufrir.

Respecto al tipo de investigación, AnderEgg Hernández (2011) filósofo, pedagógico, sociólogo y ensayista señala que se utilizará la investigación aplicada para conseguir una solución eficiente $\mathrm{y}$ debidamente fundamentada al problema específico identificado por el investigador. (p. 26)

\section{b) Método De Muestreo:}

En la investigación cualitativa las muestras son no probabilísticas, por criterio del investigador, en el muestreo no probabilista la muestra extraída no será representativa, ya que no todos los sujetos de la población tienen la misma probabilidad de ser elegidos, así mismo, se analizará expedientes y sucesos sobre el cual se recolectarán los datos, tal como lo señala Huamanchumo y Rodríguez (2015). Siendo que el presente estudio está dirigido a investigar la necesidad de los enfermos terminales de acudir a la eutanasia, por lo que únicamente la investigación se dirige a ellos, teniendo una muestra representativa y que guarde relación con el objeto de estudio.

\section{c) Escenario de estudio:}

En ese sentido como escenario de estudio para el presente trabajo de investigación se ha elegido el distrito Judicial de 
Cercado de Lima en cuanto a las entrevistas a abogados especialistas en Derecho Constitucional y médicos, ya que considero que es el lugar donde se concentra mayor índice de profesionales capaces de brindarme sus conocimientos respecto al mi investigación, así mismo se elige este distrito por ser un lugar donde se puede obtener información relevante que permita cumplir que los sujetos de esta investigación debido a la cantidad de hospitales donde se concentran los pacientes con enfermedades terminales, siendo el objeto de estudio la despenalización de la eutanasia u Homicidio Piadoso.

Para Veliz (2009), señala que cuando se trata de identificar el escenario de estudio, el investigador debe identificar cuál es el lugar más idóneo para realizar su trabajo, así como, identificar debidamente el objeto y sujetos a estudiar. (p. 194)

\section{Rigor científico}

Para los estudiosos de la metodología de investigación los asuntos de rigor relacionados con la calidad de investigación es un asunto central. El objetivo de la presente investigación analizar asuntos relacionados con el instrumento, el trabajo de campo, el análisis, el muestreo teórico, la saturación teórica y la integridad del investigador. Se tratan los dos conceptos de Validación y confiabilidad, para darle objetividad al presente artículo.

Por lo que en el presente trabajo de investigación ha sido confiable porque se entrevistará a especialistas en derecho constitucional y penal, quienes con sus conocimientos ayudaran al desarrollo de esta investigación, con la finalidad de lograr la despenalización del homicidio piadoso y la aplicación de la eutanasia en los pacientes diagnosticados con enfermedades terminales, cuya enfermedad les causa dolores insoportables, menoscabando su derecho a la salud y a la dignidad, cuyo instrumento han sido validados por expertos en Metodología de la Investigación Científica.

\section{d) Aspectos éticos}

Respecto a los aspectos éticos la información obtenida de personas entrevistadas, se mantendrá en absoluta reserva de dicha información, para ser 
analizada en forma objetiva, toda vez que la información obtenida es por parte de médicos especialistas y de abogados que litigan en el ámbito de los derechos, quienes señalan que el sufrimiento no es solo para los pacientes sino para la familia, conforme lo sostiene los metodólogos Huamanchumo y Rodríguez (2015), quienes señalan que la construcción de la realidad debe ser planificada y argumentar e interpretar los resultados en forma objetiva, y de igual forma utilizar el estilo APA versión 2017, citando los autores de la diversas fuentes bibliográficas.

\section{RESULTADOS}

\section{a) Recolección de datos}

En el presente capitulo realizare la explicación de los resultados obtenidos a través de los instrumentos de recolección de datos utilizados, donde se tomó en cuenta los objetivos y respuestas de la presente investigación, los mas resaltantes.

\section{b) Análisis cualitativo de los datos}

Por lo que en la presente investigación se usara la entrevista, a fin de conocer si es posible modificar nuestras normas, tomando en cuenta la necesidad de los enfermos terminales, así como sus testimonios mediante el cual manifiestan su voluntad de acogerse a la eutanasia.

Para Rodríguez (2008) las técnicas e instrumentos de recolección de datos son los procedimientos encargados para la recolección de información

\section{c) Presentación de los datos}

A continuación, se pasará a delinear los resultados de la investigación.

\section{Descripción de los resultados de} la técnica de Análisis de fuente documental

a) Respecto al Objetivo General: Analizar de qué manera la Despenalización de la eutanasia como derecho a la vida digna favorece a los enfermos terminales en Cercado de Lima 2019.

\section{Sentencia T-970/14 del Exp. $\mathbf{N}^{0} \mathrm{~T}$ -}

\subsubsection{Bogotá, D.C. (2014)}

En el presente documento materia de análisis para justificar el objetivo general, fue la sentencia T-970/14 del Exp. $\mathrm{N}^{\circ} \mathrm{T}$ - 
4.067.849 Bogotá, D.C. (2014) , acción de tutela contra EPS en procura de los derechos fundamentales el estado tiene como deber Constitucional la protección de la vida siendo dicho deber compatible con los otros derechos tales como la dignidad y la autonomía, en ese sentido dicho deber cede ante los enfermos terminales y prevalece la autonomía y dignidad de cada paciente que de manera consciente manifiesta su deseo de morir. Mediante la sentencia que se analiza finalmente la Corte Constitucional de Colombia ordena al Ministerio de Salud emitir una directriz mediante la cual se sugiere a los médicos un protocolo que se debatirá entre expertos de distintas disciplinas y que servirá como referente para los procedimientos del derecho a morir dignamente, así mismo, exhorto al Congreso a regular el derecho fundamental a morir dignamente.

Análisis, respecto a que si la despenalización de la eutanasia como derecho a la vida digna favorece a los enfermos terminales en Cercado de Lima - 2019, se ha señalado que si bien es cierto es deber del estado velar por la vida de las personas, tratándose de enfermos terminales, quienes ya no tienen la posibilidad de gozar o de recurar su salud, por el contrario cada día su salud se deteriora causándoles dolor insoportable y sufrimiento, el estado tienen no solo el deber, sino, la obligación de hacer valer el derecho a la dignidad de las personas por lo que si la muerte va llegar poco a poco y causando sufrimiento, la despenalización del homicidio piadoso (eutanasia) conlleva a que el enfermo terminal escoja entre morir dignamente o vivir un tiempo más pero con sufrimiento, se puede apreciar que para la legislación colombiana prevalece el derecho a la dignidad ya que ordena a su legislativo a regular el derecho a morir dignamente como un derecho fundamental.

b) Objetivo Especifico 1: Determinar si el homicidio piadoso vulnera el derecho a la libertad de las personas diagnosticadas con enfermedades terminales.

A fin de determinar el objetivo específico $\mathrm{N}^{\circ} 1$ se analizó la Sentencia $\mathrm{N}^{\circ} \mathbf{T}-493 / 93$ del Exp. T-16779, Bogotá D.C. (1993) cuyo tenor literal es como sigue: El derecho a la libertad de la persona para tomar decisiones que se ejerce dentro del 
marco del respeto del orden jurídico y de las demás personas, se encuentra dentro de los derechos fundamentales amparados por la Constitución, entiéndase que el derecho al libre desarrollo lo tienen toda persona para actuar o no según su arbitrio. Análisis: En ese sentido es evidente que el hecho que el homicidio piadoso se encuentre tipificado dentro del Código Penal, vulnera el derecho a la libertad de las personas toda vez que, no les permite llevar a cabo su deseo de culminar los últimos días de su vida con dignidad, por lo que son objetos de sufrimiento por la enfermedad que los aqueja, se tiene que entender que el acto de solicitar la eutanasia no es un acto de suicidio, sino de liberación del dolor y sufrimiento, se entiende que toda persona quisiera vivir con buena salud y de llegar el momento de su muerte que sea de manera natural y sin sufrimiento, sin embargo a tener una enfermedad terminal, tal como su nombre lo dice, TERMINAL, ésta acabará con la vida del paciente en cualquier momento, por lo que, el paciente debería tener la libertad de decidir ese momento sin que llegue a sufrir más de lo necesario a causa de su enfermedad.

c) Objetivo Especifico 2: Demostrar que el homicidio piadoso afecta la dignidad de las personas con enfermedades terminales.

Para demostrar el cumplimiento del objetivo específico $\mathrm{N}^{\circ} 2$ se analizó la Sentencia $\mathrm{N}^{\circ}$ C-239/97 del Exp. D-1490, Santa fe de Bogotá (1997), cuyo tenor literal es como sigue: El estado no puede oponerse a la decisión del enfermo terminal que no desea seguir viviendo, a causa de los sufrimientos y dolores insoportables que le causa su enfermedad y que solicita expresamente que lo ayuden a morir, ya que dichos sufrimientos no le permiten llevar una vida digna, por lo que si un enfermo terminal que se encuentra dentro de las condiciones establecidas en el art. 326 del Código Penal (Colombiano) considera que su vida debe terminar, porque la juzga incompatible con su dignidad, en ejercicio de su derecho a la libertad puede proceder sin que el Estado pueda oponerse a su designio. Análisis: De lo señalado por la sentencia de la Corte Constitucional de Colombia, se puede 
determinar que efectivamente el homicidio piadoso afecta la dignidad de los enfermos terminales, entiéndase al homicidio piadoso como el acto penalizado de la eutanasia, en ese sentido, la penalidad del homicidio piadoso no permite que el enfermo terminal lleve o culmine su vida dignamente, al obligarlo a vivir con intensos sufrimientos, lo que además estaría vulnerando no solo su derecho a una vida digna si no el derecho a elegir libremente.

\section{Descripción de resultados de la técnica: Entrevista}

En la guía de entrevista se desarrolla las preguntas de acuerdo a los objetivos, por lo que, del objetivo general se extrajo tres preguntas, del mismo modo, de cada objetivo específico se realizó tres preguntas por lo que en total la guía de entrevista consta de 9 preguntas que se detallan a continuación:

\section{OBJETIVO GENERAL:}

Analizar de qué manera la Despenalización de la eutanasia como derecho a la vida digna favorece a los enfermos terminales en Cercado de Lima $-2019$
Respecto al objetivo general se plantearon las siguientes preguntas:

1. Explique usted ¿De qué manera la despenalización de la eutanasia como derecho a la vida digna favorece a los enfermos terminales en Cercado de Lima - 2019?

Herencia (2019) menciona que, de existir un procedimiento para la eutanasia, el enfermo no se expondría a dolores insoportables, así mismo el enfermo terminal podría programar y manifestar su voluntad en todos sus asuntos, así como protegerse del encarnizamiento o ensañamiento terapéutico. Flores (2019) menciona que la despenalización de la eutanasia favorece a los enfermos terminales como una forma de consolidar su derecho a la libertad y dignidad humana. Villar (2019) menciona que la despenalización de la eutanasia favorecería al paciente terminal calmando el dolor y sufrimiento que padece, sin embargo, señala que el procedimiento de la eutanasia también le causa dolor. Tapia (2019) señala que la despenalización de la eutanasia no favorece al enfermo terminal, porque se trataría al enfermo como un objeto. Pérez 
(2019) manifiesta que no es necesaria la despenalización de la eutanasia, toda vez que, los ordenamientos jurídicos que permiten dicha práctica, se fundan en el derecho de las personas a la manifestación de su voluntad y autonomía personal del ciudadano para ponerle fin a su vida, en ese sentido para Pérez, el enfermo que quiere dejar de vivir debería suicidarse y así no necesitaría de terceras personas. Custodio (2019) señala que el amor a la vida debe realizarse cuando gozamos plenamente de ella y no sufrir tratando de aferrarnos a ella cuando ya no se puede, es decir cuando no gozamos de salud y tenemos conocimiento de que no se la va recuperar, así mismo involucra al sentimiento y deseos de los familiares del paciente. Bernal (2019) considera que la despenalización de la eutanasia debe tener un grado mayor de satisfacción que otros bienes jurídicos protegidos por el estado, es decir debe prevalecer la dignidad de la persona frente al derecho a la vida.

De los 7 entrevistados, 6 consideran que la eutanasia favorece a los enfermos terminales toda vez que, les permite decidir darle fin, a sus intolerables sufrimientos provocados por su enfermedad, sin embargo, 1 de ellos señala que, no se debería legalizar la eutanasia, ya que si el enfermo terminal desea dejar de vivir debería suicidarse y para ello no necesita de la intervención de una tercera persona.

2. ¿Qué criterios se debería tener en cuenta para que la despenalización de la eutanasia no resulte inconstitucional?

Herencia (2019) menciona que principalmente se debe tener en cuenta la manifestación de la voluntad del paciente, además de que ningún miembro de la familia se oponga a dicha decisión, que la enfermedad sea dolorosa en agonía, es decir que le cueste más, vivir que morir, que tanto el paciente como los familiares estén informados sobre el procedimiento médico y que la autorización a dicho procedimiento sea dada por un Juez. Flores (2019) menciona que se debe tener en cuenta principalmente la dignidad humana, el derecho a la vida sana en sus diversas modalidades como la moral. Villar (2019) considera que se debería modificar la constitución respecto a la vida y al homicidio. Tapia (2019) 
coincide con el comentario anterior al señalar que se debe modificar la constitución. Pérez (2019) señala que se debe modificar la constitución en cuanto a lo que se refiere al derecho a la vida, integridad física y psíquica, defensa de la dignidad de la persona, bienes jurídicos que son protegidos por los Art. 1 y 2 de nuestra carta magna, así como derogar el homicidio piadoso. Custodio (2019) considera que debe tomarse en cuenta el deseo del paciente, el amor de la familia hacia el paciente, la mejora de los derechos del paciente, verificar los artículos de la Constitución y hacer participar a la iglesia católica. Bernal (2019) señala que debe existir una comisión médica que corrobore la enfermedad incurable, además de tener en cuenta la voluntad del actor y un testigo que corrobore la voluntad del autor.

De los 7 entrevistados, 3 consideran que principalmente se debe tener en cuenta la manifestación de la voluntad del paciente a acogerse al procedimiento de la eutanasia, así como tomar en cuenta la decisión de los familiares quienes son parte de este proceso. 3 de ellos señalan que se debe modificar la Constitución en lo referente al derecho a la vida y como consecuencia la derogación del homicidio piadoso, 1 de los entrevistados considera que debe existir una comisión médica quien determine y de fe que el paciente efectivamente padece de una enfermedad incurable dicha comisión médica sería quien determina que paciente está apto para acogerse a la eutanasia.

3. ¿Considera Ud. que el desarrollo de la vida digna implica también morir dignamente? ¿Por qué?

Herencia (2019) menciona que sí, porque la vida es un derecho, no un deber por lo que, se debe considerar que todo ser humano busca una vida digna y busca poder desarrollarse como persona, lo que se vería afectado por una enfermedad la cual haga que sienta que su vida es insoportable, por lo que tiene derecho a desarrollar una muerte digna. Flores (2019) menciona que, así como gozamos del derecho a la vida, a la salud y otros derechos, la dignidad es un derecho fundamental, lo que implica una muerte justa, sin causar dolor a la familia. Villar (2019) señala que sí, porque morir dignamente es consecuencia del 
desarrollo de una vida digna. Tapia (2019) señala que sí porque una vida digna y sana termina dignamente. Pérez (2019) considera que no, señala que morir dignamente es la oportunidad de brindarle a una persona una muerte asistida para que no sufra dolor o padecimiento, sin embargo, señala que, en su condición de médico, cuando un paciente padece una enfermedad en la que ya no existe tratamiento posible, se recomienda a los familiares dar mejor calidad de vida hasta que llegue el momento del deceso natural del paciente. Custodio (2019) afirma que sí, toda vez que, morir dignamente no quiere decir acabar con tu vida, morir dignamente es que el día de tu muerte te encuentres rodeado de tus seres queridos, en el lugar que tú decides y al fin puedas descansar de sin dolor. Bernal (2019) considera que sí, porque la dignidad es el fin supremo de la Constitución, así mismo en el principio de jerarquización existen derechos fundamentales que cuentan con una jerarquía definitiva y abstracta.

De los 7 entrevistados, 6 consideran que sí, el desarrollo de la vida digna implica también morir dignamente toda vez que, vivir en una enfermedad incurable y que conlleva a dolores insoportables afecta el desarrollo de nuestra vida digna, además consideran que la dignidad como fin supremo de nuestra Carta Magna tiene el privilegio de jerarquización frente a los otros derechos que ampara la Constitución, 1 de los entrevistados señala que no, porque como médico recomienda a los familiares darle calidad de vida a sus pacientes mientras espera el deceso natural del enfermo terminal.

\section{OBJETIVO ESPECIFICO 1:}

Determinar si el homicidio piadoso vulnera el derecho a la libertad de las personas diagnosticadas con

\section{enfermedades terminales.}

Frente al objetivo especifico 1 se propusieron las siguientes preguntas:

4. ¿Considera Ud. que el homicidio piadoso vulnera el derecho a la libertad de elegir de los enfermos terminales? ¿Por qué?

Herencia (2019) señala que el homicidio piadoso o eutanasia no vulnera el derecho a la libertad de los enfermos terminales, por el contrario, les da la libertad de elegir como y cuando dentro de los procedimientos que se establezcan, 
terminar con su vida, manifestando $\mathrm{y}$ dejando todos sus asuntos en orden, los que en muchas ocasiones no lo hacen debido al ensañamiento terapéuticomédico. Flores (2019) señala que los seres humanos en condiciones de vida infrahumana, no pueden elegir seguir con vida y que si se vulnera el derecho de elegir su consecuencia. Villar (2019) señala que todo homicidio vulnera la libertad de las personas. Tapia (2019) coincide que todo homicidio vulnera la libertad. Pérez

(2019) considera que sí se vulnera la libertad de las personas porque lo que quieren es ponerle fin a su vida, aunque señala que no existe piedad en quitarle la vida de una persona enferma terminal o no, si se estaría vulnerando el derecho del enfermo a la libertad de elegir. Custodio (2019) señala que no, que toda persona tiene derecho a la vida, a una vida sin sufrimiento ni dolor y los pacientes terminales deberían ser los primeros en ser beneficiados. Bernal (2019) considera que, si se vulnera el derecho a la libertad de los enfermos terminales porque de acuerdo a lo señalado por el Tribunal Constitucional, el derecho a la dignidad es el fin supremo del estado, y el homicidio piadoso no permite que los enfermos terminales decidan darle fin a su vida dignamente.

De los 7 entrevistados 5 manifiestan que sí se estaría vulnerando el derecho a la libertad de los enfermos terminales, toda vez que, no dejarlos elegir el momento de su muerte como consecuencia de su enfermedad les exige a seguir padeciendo dolores insoportables los cuales ellos deciden no seguir tolerando, de las respuestas obtenidas se entiende que los entrevistados han considerado como pregunta si la penalización del homicidio piadoso vulnera el derecho a la libertad de los enfermos terminales por ello sus respuestas afirmativas. Mientras que dos de los entrevistados manifiestan que el homicidio piadoso no vulnera el derecho a la libertad, que por el contrario el homicidio piadoso los faculta a decidir el momento en que quieren darle fin a sus sufrimientos, se entiende de sus respuestas que ven al homicidio piadoso no como delito sino como un procedimiento eutanásico.

5. Si nuestra Carta Magna reconoce el derecho a la vida, el derecho a una 
vida digna y el derecho a la libertad ¿Considera Ud. que debería aplicarse la ponderación de derechos para el caso de pacientes diagnosticados con enfermedades terminales $y$ que manifiesten su voluntad para acogerse a la eutanasia? ¿Por qué?

Herencia (2019) señala que si se debería aplicar la ponderación de derechos siempre y cuando el paciente no sea manipulado por el sistema de salud, aunque nuestra Constitución contempla el derecho a la vida digna, no todos los peruanos tienen acceso a la vida digna, la educación digna y salud digna. Flores (2019) considera que tratándose que en nuestro país se castiga el homicidio piadoso, a pesar que tiene la misma escala que otros derechos, debe aplicarse la ponderación de derechos frente a tratamientos de enfermedades terminales. Villar (2019) señala que no hay alternativa para aliviar el dolor o sufrimiento de los pacientes terminales, por lo tanto, no debe sopesar otro derecho al derecho a la vida. Tapia (2019) señala no encontrarse de acuerdo con la eutanasia porque es ir en contra de la vida. Pérez (2019) considera que cualquier derecho de los pacientes se puede ponderar frente a otro derecho siempre y cuando dicha ponderación no vulnere el derecho fundamental a la vida y su integridad. Custodio (2019) considera que, si se debe ponderar el derecho a la dignidad frente al derecho a la vida y así respetar la voluntad y deseo del paciente, en ese sentido también se estaría ponderando el derecho a la libertad del paciente. Bernal (2019) señala que se encuentra absolutamente de acuerdo con que se pondere los derechos toda vez que, es una forma de resolver conflictos entre principios.

De los 7 entrevistados 4 consideran que se debe ponderar los derechos que favorezcan a los enfermos terminales con el fin de que logren la dignidad ansiada, toda vez que, a fin de lograr se resuelva el conflicto de derechos, la ponderación es una alternativa para evaluar la satisfacción de uno de los principios o derechos en conflicto. 3 de los entrevistados, quienes son médicos manifiestan no estar de acuerdo con la eutanasia por lo que evidentemente no consideran que debe ponderarse los derechos si se encuentran frente al 
derecho a la vida, ya que para los médicos el derecho a la vida es inviolable.

6. ¿Considera Ud. que el derecho a la libertad implica también decidir si queremos adelantar nuestra muerte, en caso de tener una enfermedad terminal y evitar los dolores insoportables y padecimientos que implican estas enfermedades? ¿Por qué?

Herencia (2019) considera que no, porque no se sabe cómo se desarrollara la enfermedad en cada paciente, por lo tanto, un paciente no debería decidir antes, sino, durante el padecimiento, en ese casi sí, se debe considerar su derecho a la libertad de elegir. Flores (2019) señala que más que elegir debe buscarse todas las alternativas que diagnostican los médicos, conforme a la Ley General de Salud, respecto al diagnóstico de la muerte. Villar (2019) considera que no, porque la razón de la libertad es preservar la vida y mejorarla en todos los aspectos. Tapia (2019) considera que no, porque en su calidad de médico señala que existen otras formas de aliviar los dolores y padecimientos. Pérez (2019) considera que el derecho a la libertad es inherente a cada persona independientemente de su estado de salud, por lo que a un paciente con enfermedad terminal le asiste la libertad de decidir si adelanta o no su muerte por sus propios medios ya sea por comisión y omisión respetando su dignidad. Custodio (2019) señala que las enfermedades terminales tienen un nivel de gravedad, si el paciente se encuentra en la última etapa considera que debería elegir evitar mayor sufrimiento. Bernal (2019) manifiesta que la libertad de una vida digna es un valor superior del ordenamiento que preexiste del orden estatal, a través de la ponderación, de presentarse conflictos entre bienes constitucionales se deberá resolver haciendo prevalecer uno de ellos, por lo que en referencia al tema en investigación el derecho a la libertad de elegir del paciente terminal, tienen que ver con el disfrute del derecho a la vida digna.

De los 7 entrevistados, 3 de ellos están de acuerdo que el derecho a la libertad también debe implicarse el decidir si queremos darle fin a nuestra vida, solo en el caso de los enfermos terminales quienes no solo le quieren dar fin a su vida si no a sus sufrimientos, manifestando además que el derecho a la 
libertad de elegir de los enfermos terminales va de la mano de su derecho a la dignidad, derechos que deben ponderarse frente al sufrimiento que les hace padecer sus enfermedades, 3 de los entrevistados considera que el derecho a la libertad de los enfermos terminales va más direccionado al derecho a preservar su vida, y que existen otros medios de aliviar los dolores que no son dar muerte. 1 de los entrevistados manifiesta que se debería tener en cuenta no solo el derecho a elegir del paciente sino lo que señala la Ley General de Salud respecto al diagnóstico de la muerte.

\section{OBJETIVO ESPECÍFICO 2}

Demostrar que el homicidio piadoso afecta la dignidad de las personas con enfermedades terminales.

A fin de demostrar el cumplimiento del objetivo especifico 2 se plantearon las siguientes preguntas:

7. Para Ud. ¿De qué manera el homicidio piadoso afecta la dignidad de las personas con enfermedades terminales?

Herencia (2019) señala que el homicidio piadoso no afecta la dignidad de los enfermos terminales, entiende al homicidio piadoso como eutanasia y no como delito, por lo que, explica que el homicidio piadoso podrá ayudar al paciente a decidir si desea o no someterse a terapias dolorosas o solo mantener una calidad de vida hasta un debido tiempo. Flores (2019) menciona afecta la dignidad humana por la forma como su dignidad moral y emocional tanto suya como la de sus familiares se ve afectada, además de que el homicidio piadoso restringe su derecho a la libertad de elegir entre uno u otro derecho. Villar (2019) para Villar, en la medida que no se respeta su vida, tampoco se estaría respetando su dignidad. Tapia (2019) señala que el homicidio piadoso afecta la dignidad de los enfermos terminales porque se trata a las personas como objetos, lo que daña su dignidad. Pérez (2019) considera que una muerte provocada no puede considerarse digna ya que implica el cese de la vida en forma no natural. Custodio (2019) considera que si los derechos están establecidos el homicidio piadoso no afectaría la dignidad de las personas tanto, ética, moral y religiosamente. Bernal (2019) 
considera que, si se afecta la dignidad de los enfermos terminales porque, se estaría dando mayor importancia al derecho a la vida de los pacientes diagnosticados con enfermedades incurables, desplazando de esta manera el derecho a la libertad de elegir una vida digna.

De los 7 entrevistados, 4 de ellos considera que el homicidio piadoso afecta la dignidad de las personas, en el sentido de que no les permite decidir terminar su vida con dignidad al estar obligados a vivir con dolor y en pésimas condiciones de salud, además de afectar no solo físicamente al paciente, sino que, al ver el sufrimiento de sus familiares, les afecta psicológicamente, ya que se sentirían una carga para su familia. Por otro lado 3 de los entrevistados, consideran que no se estaría afectando el derecho a la dignidad porque una muerte provocada por un tercero no puede considerarse digna.

8. Cuándo nos referimos a un enfermo en fase terminal ¿Podríamos presumir que goza del derecho a una vida digna? ¿Por qué?

Herencia (2019) manifestó que no se puede hablar de vida digna, toda vez que, en cuanto se quiebra la salud, en el caso de enfermedades terminales, no puede ser tolerable aguantar la vida. Flores (2019) señala que no, porque el derecho a una vida digna significa vivir una calidad de vida tanto interna como externa, dentro de la moral, con disfrute y goce del mundo exterior, lo que una enfermedad terminal no permite. Villar (2019) señala que toda vida es digna cualquiera sea su forma, por lo tanto, el enfermo terminal goza de vida digna. Tapia (2019) señala que toda persona en general goza de vida digna, el homicidio en cualquiera de sus formas, sería desperdiciar la vida. Pérez (2019) identifica como vida digna la capacidad de nacer, desarrollarse, reproducirse y morir, desde aquella perspectiva considera que los enfermos terminales gozan de vida digna ya que estarían cumpliendo con el ciclo de la vida.

Custodio (2019) considera que los enfermos terminales, si gozan del derecho a la vida digna, porque las enfermedades tienen fases y si se llegó a una fase terminal es porque se ha pasado las anteriores fases tratando de lograr la recuperación. Bernal (2019) para vernal los enfermos terminales no gozan del derecho a la vida digna, toda vez que, 
según la Declaración Universal de Los Derechos Humanos de las Naciones Unidas, todo individuo tiene derecho a la vida, pero nadie será sometido a torturas, ni penas o tratos crueles, por lo tanto, la vulneración de este, afecta la vida digna.

De los 7 entrevistados 4 de ellos quienes se encuentran ligados al sistema de salud, consideran que el simple hecho de que exista vida se puede hablar de dignidad, siendo el homicidio en cualquiera de sus formas quien vulnera la dignidad y la vida de las personas sean enfermos terminales o no, de otro lado, 3 de los entrevistados consideran que no se puede hablar de vida digna cuando se padece dolores intolerables y existe el quiebre de la salud, conllevando a una enfermedad cuya consecuencia es la muerte, así mismo señalan que el derecho a la dignidad está protegido por las normas internacionales, las que prohíben las torturas y tratos inhumanos.

9. Frente al presente problema del homicidio piadoso, ¿Cuál serían las alternativas a fin de que el médico o especialista no sufra las penalidades que señala el art. 112?
Herencia (2019) considera se debe derogar el Art. 112 del Código Penal. Flores (2019) menciona que la regulación de la eutanasia sería una herramienta muy importante para que el médico tratante se libere de la responsabilidad establecida en el Art. 112 del Código Penal. Villar (2019) considera que no se debe realizar el homicidio piadoso. Tapia (2019) considera que para que el médico no sufra las penalidades del Art. 112 del Código Penal, no debe practicar el homicidio piadoso. Pérez (2019) considera que habiendo prohibición de practicar el homicidio piadoso, el médico solo puede inhibirse de realizar dicha práctica, sin embargo considera como alternativa la modificación de la Constitución respecto al derecho a la vida y como consecuencia derogar las normas que prohíban el homicidio piadoso, así mismo considera como tercera alternativa que los familiares del paciente realicen el pedido de eutanasia ante un ente judicial, solicitando se exonere al médico de cualquier responsabilidad. Custodio (2019) señala que, si los derechos se encuentran claro y establecidos, ningún profesional tendría que verse involucrado, 
así mismo como consecuencia de ello los colegios profesionales tendrían que modificar sus estatutos o normas afín de evitar alguna sanción tanto penal o administrativa. Bernal (2019) Considera que se debería analizar el homicidio piadoso teniendo en cuenta los presupuestos del aborto terapéutico.

De lo 7 entrevistados, 4 consideran que se debe modificar tanto la Constitución Política como el Código penal respecto al homicidio piadoso. 3 consideran que no se debe despenalizar el homicidio piadoso, en su calidad de médicos respetan el derecho a la vida.

\section{TESTIMONIO DE ANA ESTRADA,} diagnosticada con polimiositis, enfermedad autoinmune, progresiva y degenerativa que atrofia e inflama los músculos causando pérdida de fuerza.

En su blog respecto a la muerte digna señala lo siguiente: "Que necesito tener la certeza de poder decidir cuándo y cómo querer partir para poder ser feliz. Les cuento: según las estadísticas de estos organismos, afirman que solo una cuarta parte de los inscritos para recibir la dosis letal en el momento que ellos decidan, han llevado a cabo el suicidio asistido. Y, más bien, la gran mayoría de ellos nunca lo usaron y esperaron la muerte natural. ¿Comprenden lo paradójico de esto? Que mientras no tenga el poder de mi libertad seguiré viviendo presa en un cuerpo que se está deteriorando cada minuto y que me atará a mi cama conectada las 24 horas al respirador y empezarán las úlceras en la piel que no son otra cosa que heridas que se expanden y profundizan hasta que se logra ver el hueso. Esas heridas supurarán pus y olerán a podrido y el tejido se va a necrosar. Pero eso será solo el comienzo de sendas infecciones y más medios invasivos y amputaciones y no moriré. Ese infierno será eterno y, repito, mi mente estará completamente lúcida para vivir cada dolor en una cama de hospital sola y queriendo morir",

"Es difícil hablar de la muerte. Es aún más difícil cuando se trata de alguien que necesita tener la certeza de morir para poder vivir. El suicidio asistido en el Perú es ilegal, vivo en un Estado que me quita la libertad, y una vida sin libertad no es vida porque mi cuerpo le pertenece a este Estado.

\section{DISCUSION} y CONCLUSIONES 
Para este artículo se determinó considerar los resultados obtenidos en los trabajos previos, los conceptos del marco teórico, las entrevistas realizadas y el análisis de fuente documental, estudiados en el presente artículo, los mismos que quedaron de la siguiente forma:

a-Respecto al objetivo jurídico General del presente artículo que es Analizar de qué manera la despenalización de la eutanasia como derecho a la vida digna favorece a los enfermos terminales en Cercado de Lima - 2019.

La despenalización de la eutanasia favorecería el desarrollo de una vida digna en los enfermos terminales, toda vez que, les permitiría acogerse a la eutanasia $y$ dar fin a sus graves padecimientos físicos o psicológicos.

Los especialistas entrevistados, la mayoría de ellos consideran que de conseguir que se apruebe la despenalización de la eutanasia se estaría logrando que los enfermos terminales vean que se respeta su derecho a la vida digna, siendo que la dignidad es la autodeterminación responsable de la vida y por ello se pretende su respeto por parte de los demás, en consecuencia, los enfermos terminales, tienen derecho al respeto de su dignidad.

Los entrevistados, señalan que para que la despenalización del homicidio piadoso no resulte inconstitucional deberá tenerse en cuenta que no se vulnere el derecho a la dignidad de los enfermos terminales, siendo que el hecho de que un paciente se encuentre postrado en una cama de hospital dependiendo de un aparato para vivir, no resulta digno, por lo que, al despenalizar la eutanasia, se estaría cumpliendo con lo establecido en nuestra carta magna, respecto a la dignidad como fin supremo del estado y la sociedad.

Asimismo, la mayoría señalan que el desarrollo de la vida digna implica también morir dignamente ya que el simple hecho de tener una enfermedad terminal afecta el desarrollo de la vida digna, siendo el respeto a la dignidad de la persona es el fin supremo de la sociedad y el Estado, tal como lo señala nuestra Constitución Política, las normas complementarias deberían regular el derecho a una muerte digna en los casos de los enfermos terminales.

En consecuencia, los resultados coinciden con lo dicho por García (2007) respecto a 
la vida digna que las personas poseen como principal derecho, para llevar una vida digna debe existir un equilibrio armónico entre la mente, el bienestar físico y psíquico, quedando demostrado que la eutanasia es el medio para lograr que sea efectivo el fin supremo de la sociedad y el estado, que es el respeto de la dignidad de las personas, en el presente caso de las personas con enfermedades terminales, toda vez que, les permite que tengan el derecho a decidir dejar de sufrir los padecimientos de su enfermedad

En consecuencia, de los resultados actuales respecto a la despenalización de la eutanasia se evidencia que la despenalización de la eutanasia favorece el desarrollo de la vida digna de los enfermos terminales, por lo que, considero que debe ser regulado en nuestro ordenamiento jurídico.

b)-El Objetivo Especifico 1 de la presente investigación es: Determinar si el homicidio piadoso vulnera el derecho a la libertad de los enfermos terminales.

Al despenalizar el homicidio piadoso estaríamos respetando el derecho a la libertad y autonomía del enfermo terminal para que decida sobre el fin de sus días.
Los entrevistados, la mayoría manifiestan que sí se está vulnerando los derechos de los enfermos terminales en este caso el derecho a la libertad y autonomía que poseemos todas las personas sin excepción, toda vez que, no les permite decidir si darle fin a su vida, no solo desde la perspectiva del homicidio o el suicidio, sino, lo consideran darle fin a los sufrimientos causados por la enfermedad que los aqueja.

Los entrevistados, la mayoría considera que debe aplicarse la ponderación de derechos en los casos como la eutanasia, en el caso en investigación, no se aplica la eutanasia porque se considera que se estaría vulnerando el derecho a la vida, sin embargo, estamos hablando de personas que viven con intensos dolores provocados por su enfermedad que además es terminal, lo que quiere decir que, la consecuencia de esa enfermedad finalmente será la muerte, entonces porque no permitir que ejerzan su derecho a la libertad y autonomía para hacer respetar su dignidad como personas, ya que ellos consideran no digno vivir en esas condiciones. 
Los entrevistados, la mayoría considera que el derecho a la libertad también debe implicar el decidir cuándo darles fin a los padecimientos sufridos por su enfermedad terminal y en consecuencia a su vida, teniendo en cuenta además de lo señalado en la Ley General de Salud respecto al diagnóstico de la muerte.

De las entrevistas podemos decir que, el homicidio piadoso como delito en nuestro Código Penal, vulnera el derecho a la libertad de las personas ya que, al no dejarlos decidir sobre el fin de su vida como consecuencia de su enfermedad terminal, los obliga a vivir en sufrimiento, vulnerado no solo el derecho a la libertad sino además, el derecho a la dignidad de las personas, y la prohibición de lo señalado por la Declaración Universal de los Derechos Humanos que prescribe que nadie será sometido a torturas, penas, o tratos crueles e inhumanos o degradantes, considerando que los enfermos terminales son sometidos a tratos inhumanos es evidente que no solo se está violando la norma Constitucional.

Sentencia de la Corte Constitucional de Colombia STC T-493-93 que señala:
El derecho al libre desarrollo o desenvolvimiento de la personalidad, o de libertad de opción y de toma de decisiones de la persona, ejercido dentro del marco del respeto de los derechos de los demás y el orden jurídico, es un derecho constitucional fundamental, que se vulnera con la penalización de la eutanasia. Así mismo me baso en el siguiente fundamento legal:

CONSTITUCIÓN POLITICA DEL PERÚ:

Art. 2.24 Derecho a la libertad y a la seguridad personal:

Literal h: Nadie debe ser víctima de violencia moral, psíquica o física, ni sometido a tortura o a tratos inhumanos o humillantes.

En consecuencia, los resultados actuales reafirman que el homicidio piadoso efectivamente está violando la libertad de los enfermos terminales, ya que, de lograr su despenalización, la eutanasia les permitiría elegir entre seguir viviendo bajo el sufrimiento de su enfermedad, o darles fin a esos padecimientos y terminar su vida con dignidad ejerciendo su derecho de libertad. 
El Objetivo Especifico 2 de la presente investigación: Demostrar que el homicidio piadoso afecta la dignidad de las personas con enfermedades terminales.

Los entrevistados la mayoría considera que efectivamente el homicidio piadoso vulnera la dignidad de las personas ya que los obliga a continuar en situación inhumana sufriendo padecimientos innecesarios que la eutanasia podría aliviar.

Los entrevistados, la minoría considera que no se puede hablar de vida digna cuando se padece de una enfermedad terminal cuya consecuencia además de los insoportables dolores es la muerte.

Los entrevistados manifiestan que debe constar expresamente el derecho a morir dignamente en nuestra constitución, con ello se podrá modificar el Código Penal y derogar su Art. 112 el cual penaliza el homicidio piadoso o en su defecto subsumirlo a una modalidad del delito de homicidio si este es realizado por una persona que no es el médico autorizado para realizar el procedimiento de la eutanasia.
De las entrevistas podemos decir que, la mayoría de los entrevistados consideran el homicidio piadoso afecta la dignidad de las personas por lo que la modificación del Código Penal derogando el homicidio piadoso, sería la alternativa para que estas personas vean aliviado su dolor y sientan que se les respeta la dignidad.

En consecuencia, de los resultados obtenidos se infiere que existe coincidencia en con lo señalado en la Sentencia C-239/97 que señala que los sufrimientos y padecimientos consecuentes de una enfermedad terminal, no les permite tener una vida digna, por lo que, la modificación de nuestro Código Penal en su artículo 112 podría ser posible siempre se encuentre o se agregue en la Constitución Política del Perú el derecho a morir dignamente y se regule la eutanasia sin dejar vacíos en la Ley.

En el presente Artículo se discute, que el Homicidio Piadoso, no debe ser penalizado, no solo por un sustento Jurídico, sino de salud, de dignidad, de libertad, de calidad de vida que tanto defienden los países democráticos, que al colocar en la Balanza existe una gran 
diferencia entre la Verdad Jurídica versus la Verdad real, porque el derecho no debe ser determinante, es solo un indicador inconsistente.

En cuanto a las conclusiones señalamos que, de acuerdo a la identificación del problema, la revisión de los antecedentes, del análisis e interpretación del marco teórico y del trabajo de campo realizado, se ha llegado a las siguientes conclusiones:

Primero: Se ha determinado que la despenalización del homicidio piadoso favorece al desarrollo de una vida digna a los enfermos terminales, toda vez que de llegar a aprobarse la eutanasia estos tendrían una alternativa para decidir si seguir viviendo con sufrimiento o ponerle fin a sus padecimientos y lograr el descanso, siendo contradictorio e inconsistente jurídicamente el Art. 112 del Código Penal de Perú, concordante con nuestra Constitución Política, Declaración de los Derechos Universales. Quedando demostrado de acuerdo a los resultados obtenidos de los testimonios, de las entrevistas a especialistas incluido médicos, quienes son testigos directos de los padecimientos de los enfermos terminales, tal como es el caso de Ana Estrada, que ha recurrido a la Defensoría del Pueblo para que defienda los Derechos Fundamentales, quien solicita acogerse al beneficio de la despenalización del homicidio piadoso, sustentando que la eutanasia es el medio para lograr el desarrollo de la dignidad de los enfermos terminales.

Segundo: Se ha analizado si el homicidio piadoso vulnera el derecho a la libertad de los enfermos terminales, concluyendo que efectivamente la penalización de la eutanasia a través del homicidio piadoso vulnera el derecho a la libertad y autonomía de las personas al no poder decidir libremente y ser obligados a continuar viviendo bajo el sufrimiento causado por su enfermedad, siendo que la Constitución Política Art. 2 inciso 1-. A la vida, a su identidad, a su integridad moral, psíquica y física y a su libre desarrollo y bienestar. El concebido es sujeto de derecho en todo cuanto le favorece, por tanto le favorece no sufrir, bajo ninguna condición porque se sustentan en mi bienestar.

Tercero: De acuerdo a los resultados de las entrevistas realizadas a especialistas y 
del análisis de las fuentes documentales, se ha determinado que el homicidio piadoso, que se encuentra tipificado en el Art. 112 del Código Penal, vulnera el derecho a la dignidad, derecho que se encuentra amparado no solo por nuestra Constitución Política en su Art. 1, sino por normas internacionales, toda vez que, el homicidio piadoso no permite que los enfermos terminales desarrollen o ejerzan su derecho a la vida digna, prohibiéndoles acogerse a la eutanasia, verificando de esta manera el segundo objetivo jurídico específico.

El presente Artículo, respecto al HP, se debe ponderar; el Derecho que tiene toda persona, Derecho a la Vida, Derecho a la Libertad, Derecho a la Dignidad. Pero el Derecho como tal a la fecha no soluciona los problemas sociales, porque tradicionalmente solo es un conjunto de Normas, siendo la verdad un conjunto de hechos sociales contradictorios al Derecho, por tanto la HP, es menos gravoso para la víctima, Doctrina sustentado por Luiguí Ferrajoli y Robert Alexis.

En Perú, como ha sucedido en España, existen testimonios de ciudadanos que han recurrido al TC, a la $\mathrm{CIDH}$, máximas autoridades para hacer prevalecer su Derecho de vivir Dignamente y quitarse la vida por ser su derecho a elección, no son escuchados, porque la Industria Millonaria de los Fármacos, bajo la protección del modelo democrático. Caso: Ana Estrada, sufre 15 años de polimiositis y ha solicitado a la Defensoría del pueblo Perú, quien solicita el cese de su vida, por estar 20 horas en cama y 4 en silla de ruedas ya no puede moverse, su testimonio es desgarrador. ¿Pero quién se opone?, no es una Constitución, no es Código Penal, es un poder lleno de interés económicos.

Habiendo identificado las conclusiones a las que lleva el presente artículo, se plantea las siguientes recomendaciones:

Primero: $\mathrm{Se}$ recomienda la Despenalización del Homicidio Piadoso, por ser un Derecho Constitucional, y Derecho Universal de la "Liberdad". Los ciudadanos que están sufriendo puedan acudir a los países donde no es penalizado. 
Segundo: Se recomienda que debe prevalecer el Derecho a la Libertad, por Constitucional y Universal y la manifestación de la voluntad expresada sea escuchada por la Industria de los Fármacos.

Tercero: Se recomienda que el artículo 1 de la Constitución, que señala, dignidad de la persona es el fin supremo del estado y la sociedad, en ese sentido, debe primar su bienestar que es inherente a la persona.

\section{Referencias:}

-Bacon, F (1950). El avance de la ciencia, Everyman Library, Londres.

Blanco, Luis G. Homicidio piadoso, eutanasia y dignidad humana. En Revista Jurídica La Ley T. 1997-F, pp. 509-527. Buenos Aires: La Ley.

Blanco, Luis G. (2013). Homicidio piadoso. En Gil Domínguez, Andrés (Director). Muerte digna, pp. 383/403. Buenos Aires: La Ley.

- Calsamiglia Blancafort, A (2002). "Sobre la eutanasia". En VÁSQUEZ,
Rodolfo. Bioética y Derecho: fundamentos $\mathrm{y}$ problemas actuales. Editorial ITAM/FCE, México.

-Dworkin. R. (1994). Sobre la disponibilidad del individuo sobre su vida. Reino Unido.

-Ferrajoli, L. (1995). Derecho y Razón. Teoría del garantismo penal, Editorial Trotta, Madrid.

- García Toma, Víctor, 2008 Los Derechos Fundamentales en el Perú. (p. 22)

-Gil. F. (2016). Franciscano, en su obra "la prohibición de matar". España.

-Guaricaja (2011) "Proyecto de Legalización de la Eutanasia, por la protección al derecho a la vida digna, en la Legislación Ecuatoriana" (Tesis de grado) Universidad Técnica de CotopaxiEcuador.

Jacobs, Günther (1996). Sobre el injusto del suicidio y del homicidio a petición. Bogotá: Universidad Externado de Colombia. 
-Medina Frisancho, J. (2010). Eutanasia e imputación objetiva en Derecho penal, Editorial Grijley, Lima.

-Maestre y Romero (2001 "Eutanasia: Un asunto de cuidado intensivo". (Tesis de grado) Pontificia Universidad Javeriana Bogotá - Colombia.

-Martínez (2017) "Derecho a una muerte digna: La necesidad de regulación de la eutanasia y el suicidio asistido en México" (Tesis de grado) Facultad de Derecho de la Barra Nacional de Abogados - México.

Flemate (2015) "El derecho a la muerte digna en el ordenamiento jurídico México". (Tesis doctoral) Universidad de Castilla - La Mancha - España.

-Ortega (2015) En la Tesis titulada "Eutanasia: De delito a derecho humano fundamental. Un análisis de la vida a partir de los principios fundamentales de la libertad, autodeterminación, dignidad humana y más allá de la mera existencia" (Tesis de maestría) Corporación Universitaria Libre de Colombia, facultad de Derecho.
-Baca (2017) "La Eutanasia y el derecho a morir dignamente para su despenalización" (Tesis de grado) Universidad Autónoma del Perú.

-Tarrillo y Arribas plata (2017) "Razones jurídicas para la despenalización de la eutanasia en la Legislación del Perú, Año 2017" (Tesis de maestría) Universidad Privada Antonio Guillermo Urrelo.

-Flores y Padilla (2015) "La no punibilidad de la conducta del médico que realiza el homicidio a petición en nuestro ordenamiento jurídico" (Tesis de grado). Universidad Nacional de la Amazonia Peruana.

-Recoba (2015) “Análisis de la eutanasia dentro del tipo penal de homicidio piadoso en la legislación peruana" (Tesis de grado). Universidad Nacional de Piura.

-Mendoza (2014) "Eutanasia: Un ensayo de fundamentación liberal para su despenalización" (Tesis de maestría). Pontificia Universidad Católica del Perú.

-Pessini (2008); en su obra denominada "como lidiar con un paciente en fase terminal", Madrid. 
IUS ET SCIENTIA (ISSN 244-8478) 2019, Vol. 5, nº 2, pp. 111-144. DESPENALIZACIÓN DE LA EUTANASIA COMO DERECHO A LA DIGNIDAD DE LOS ENFERMOS TERMINALES EN CERCADO DE LIMA, 2019.

Delia Chávez Santamaría delia.ch.18@gmail.com y Jorge Rodríguez Figueroa, jrodriguezfig@ gmail.com Universidad César Vallejo (Perú)

Recibido: 17/09/2019. Aceptado: 10/10/2019 DOI: http://doi.org/10.12795/IESTSCIENTIA.2019.i02.06

Revista, Declaración sobre la eutanasia, en "Medicina Paliativa", 2002, pp. 37-40

Rivera (2003) Eutanasia Autonomía. (p.93)

-Marín Castán, María Luisa. "La Dignidad Humana, Los Derechos Humanos y los Derechos Constitucionales". Revista de Bioética y Derecho. Universitat de Barcelona. España. 2007.

-Roxin, Claus. Eutanasia y Suicidio. Cuestiones Dogmáticas y de Política Criminal. Granada 2011.

-Sánchez. T. (2012). Despenalización del homicidio a petición, Artículo $112^{\circ} \mathrm{del}$ código penal peruano, desde una perspectiva constitucional y penal (tesis de Magister). Universidad de Antúnez de Mayolo, Huaraz.

-Santos. G. (2015). Análisis de la despenalización del homicidio por piedad, su implicación en el ordenamiento jurídico.

-Sentencia del Tribunal Constitucional recaída en el Expediente $N^{\circ}$ 5954-2007HC/TC. Fundamento 57.
Sentencia T-970/14 del Exp. $\mathrm{N}^{\mathrm{o}} \mathrm{T}-$ 4.067.849 Bogotá, D.C. (2014)

Sentencia N ${ }^{\circ}$ T-493/93 del Exp. T-16779, Bogotá D.C. (1993)

Sentencia N C-239/97 del Exp. D-1490, Santa fe de Bogotá (1997)

URE, Ernesto J. (1959). Homicidio piadoso. En Lecciones y Ensayos, № 14, pp. 7 y ss. Buenos Aires: Facultad de Derecho y Ciencias Sociales, UBA.

\section{Libros de Metodología}

Bernal, C. (2006). Metodología de la Investigación. Segunda Edición.

Cegarra Sánchez José Metodología de la Investigación Científica y tecnología

Eyssautier de la Mora, M. (2006). Metodología de la Investigación, desarrollo de la inteligencia. (5. ${ }^{\mathrm{a}}$ ed.) México: Thomson Learning.

Galindo, L. (1998). Técnicas de Investigación en Sociedad, Cultura y Comunicación. México: Pearson Educación Latinoamérica.

Hernández, R. et al (2014). Metodología de la investigación. México: McGrawHill, 6ta Ed.

Huamanchumo y Rodríguez (2015) Metodología de la investigación en las 
organizaciones. Lima, Perú, Editorial Summy

Méndez, C. (1995) METODOLOGIA. Guía para elaborar diseños de investigación en ciencias económicas, contables y administrativas. Bogotá: Ed. McGraw-Hill.

Namakforoos y Mohammad, N. (2005). Metodología de la Investigación. (2. ${ }^{a}$ ed.). México: Limusa-Noriega Editores.

Polity, D. y Hunger, B. (2000). Investigación Científica en Ciencias de la Salud.(6. ${ }^{a}$ ed.). México: Mc Graw Hill Interamericana.

Rojas, R. (2013). Guía para realizar investigaciones sociales. (38.a ed.) Lima: editores Plaza y Valdez.

Ruiz, J. (2012). Metodología de la Investigación Cualitativa. (5. Aed.).

Bilbao: Deusto. 